\title{
A summary of recent results from the GRAPES-3 experiment
}

\author{
S.K. Gupta ${ }^{1,2, a}$ \\ 1 Tata Institute of Fundamental Research, Homi Bhabha Road, Mumbai 400005, India \\ 2 The GRAPES-3 Experiment, Cosmic Ray Laboratory, Raj Bhavan, Ooty 643001, India (On behalf of the GRAPES-3 \\ Collaboration)
}

\begin{abstract}
The GRAPES-3 experiment is a combination of a high density extensive air shower (EAS) array of nearly 400 plastic scintillator detectors, and a large $560 \mathrm{~m}^{2}$ area tracking muon telescope with an energy threshold $\mathrm{E}_{\mu}>1 \mathrm{GeV}$. GRAPES-3 has been operating continuously in Ooty, India since 2000. By accurately correcting for the effects of atmospheric pressure and temperature, the muon telescope provides a high precision directional survey of the galactic cosmic ray (GCR) intensity. This telescope has been used to observe the acceleration of muons during thunderstorm events. The recent discovery of a transient weakening of the Earth's magnetic shield through the detection of a GCR burst was the highlight of the GRAPES-3 results. We have an ongoing major expansion activity to further enhance the capability of the GRAPES-3 muon telescope by doubling its area.
\end{abstract}

\section{Introduction}

The GRAPES-3 (Gamma Ray Astronomy at PeV EnergieS-phase 3) is a high density extensive air shower (EAS) array which was designed for a precision study of the acceleration of charged particles in nature by using a number of phenomena as explained below. The GRAPES-3 experiment had a modest beginning with its first component comprising 256 plastic scintillator detectors (each $1 \mathrm{~m}^{2}$ in area) in Ooty, India in 2000 as shown schematically in Fig. 1. The array has now been expanded to about 400 detectors which are deployed in a dense hexagonal configuration with an inter-detector distance of $8 \mathrm{~m}$, in Ooty ( $2200 \mathrm{~m}$ altitude, $11.4^{\circ} \mathrm{N}$ latitude, $76.7^{\circ} \mathrm{E}$ longitude). About $25 \%$ of these detectors are at present instrumented with two fast photomultiplier tubes (PMTs) for the purpose of extending their dynamic range to $\sim 10^{4}$ particles $\mathrm{m}^{-2}$. The scintillators, the signal processing electronics, and the data acquisition systems were all designed, and fabricated in-house to minimize costs, and to optimize performance [1].

The second component of the GRAPES-3 array contains a large area $\left(560 \mathrm{~m}^{2}\right)$ tracking muon telescope [2] to measure the cosmic ray induced muon flux with high statistical precision. This high statistical precision has permitted us to probe the acceleration of muons in the atmospheric electric fields set up during major thunderstorm activity. These thunderstorm activities are also known to result in the generation of terrestrial flashes of $\gamma$-rays [3-5]. The muon telescope has also provided new information with very high sensitivity on solar flares, coronal mass ejections (CMEs), and Forbush decrease (Fd) events $[6,7]$ besides serving as a sensitive probe of the interplanetary magnetic field, and the turbulence caused in it due to the propagation of CMEs $[8,9]$.

a e-mail: gupta.crl@gmail.com
It had been shown earlier by us that the primary cosmic ray spectrum, and composition can be extracted using the muon multiplicity, and the shower size distributions of the detected EAS [10]. By using the GRAPES-3 data, this technique was employed to determine the composition of the primary cosmic rays from $30 \mathrm{TeV}$ to $1 \mathrm{PeV}$, along with a sizeable overlap with the direct measurements from balloon- and satellite-borne detectors. However, this interpretation of EAS data involved the extrapolation of hadronic interaction properties to energies beyond those available from accelerators. This necessitates modeling of high energy interactions assuming certain reasonable assumptions leading to several models of hadronic interactions which depend on the nature of underlying assumptions [11-16]. Therefore, the interpretation of the EAS data in terms of composition and energy spectrum gets coupled to the choice of the hadronic interaction models used. In GRAPES-3 data, we used the overlap in the energy with the direct measurements to identify the hadronic interaction models that had yielded composition and energy spectra consistent with the direct measurements in the overlap energy region from $300 \mathrm{TeV}$ to $1 \mathrm{PeV}$ [17].

\section{Experimental details}

To operate the GRAPES-3 EAS array at the lowest possible threshold energy, a simple 3-line coincidence of detectors is used to generate the Level-0 trigger, that acts as the fast GATE and START for the charge to digital (ADCs) and time to digital converters (TDCs), respectively. Since this trigger selects very small local showers and also larger showers whose cores land far from the area covered by the array, we require at least 12 out of the inner 127 detectors should have triggered their discriminators within $1 \mu \mathrm{s}$ of the Level-0 trigger. This Level-1 trigger with an observed EAS rate of $15 \mathrm{~Hz}$ is used to record the charge (ADC) and the arrival time (TDC) of the pulses from each detector [1]. 


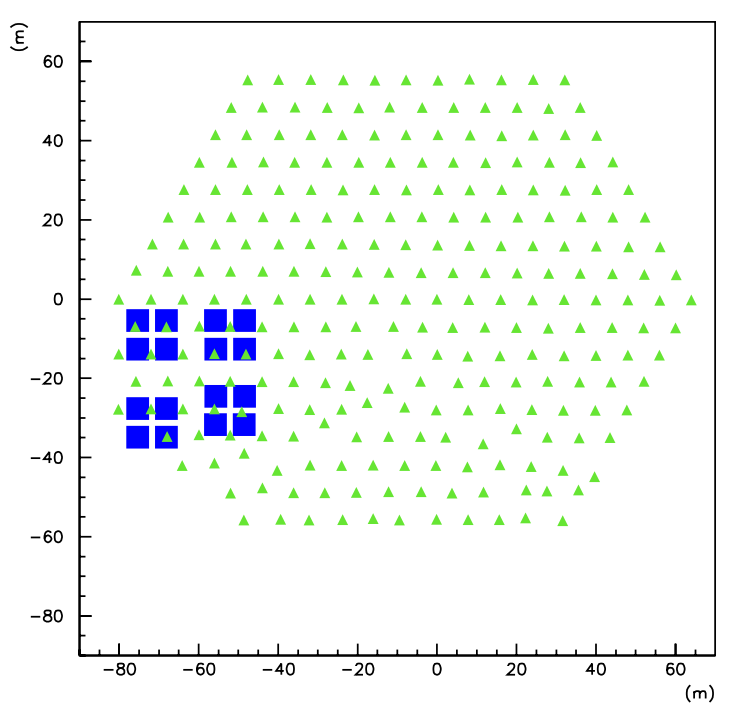

Figure 1. A schematic layout of the GRAPES-3 EAS array with 256 detectors (triangles). Sixteen squares represent a $35 \mathrm{~m}^{2}$ area muon telescope with $E_{\mu} \geq 1 \mathrm{GeV}$.

With the expansion of the array the trigger rate has now increased to $35 \mathrm{~Hz}$. The pulse charge is later converted into equivalent number of minimum-ionizing particles (MIPS) using the most probable charge for a single measured MIP. The high sensitivity of scintillator detectors allows the atmospheric radon decay products to be routinely detected during every episode of rain in Ooty.

In Fig. 1 each square shown in the lower left-side, represents a 4-layer muon telescope module with an energy threshold of $1 \mathrm{GeV}$ for vertical muons. Each layer contains 58 proportional counters (PRCs), each $600 \times 10 \times 10 \mathrm{~cm}^{3}$ in size. Thus, the sixteen modules that constitute a total area of $560 \mathrm{~m}^{2}$ of the muon telescope are shown as sixteen squares in Fig. 1. The energy threshold of $1 \mathrm{GeV}$ was achieved by placing a concrete absorber of thickness $550 \mathrm{~g} \mathrm{~cm}^{-2}$ above the bottom PRC layer. Thus, each module with a sensitive area $35 \mathrm{~m}^{2}$ consists of 232 PRCs arranged in four layers of 58 PRCs each, with alternate layers placed in orthogonal directions. Two successive layers of PRCs are separated by a $15 \mathrm{~cm}$ thick concrete layer. This four-layer configuration allows a 3-D reconstruction of each muon track to an accuracy of $\sim 4^{\circ}$. The sixteen telescope modules detect $\geq 1 \mathrm{GeV}$ muons at a rate of $4 \times 10^{9} \mathrm{~d}^{-1}$, allowing tiny variations due to atmospheric, solar, and other systematic effects to be precisely measured. Using the reconstructed muon direction the muons are segmented into nine solid-angle bins, labeled Vertical (V), North (N), South (S), East (E), West (W), South-East (SE), North-East (NE), South-West (SW), North-West (NW) covering a solid angle of $2.3 \mathrm{sr}$.

\subsection{Muon module efficiency corrections}

The daily rate of muons measured for a period of 100 days (1 Jan.-10 Apr. 2006) by modules 4 and 5 is shown in Fig. 2a, Fig. 2b, respectively. The rates are nearly the same, and display similar variation caused by the atmospheric and solar effects. However, the ratio of their rates shown in Fig. $2 \mathrm{c}$ displays a significantly smaller $(<0.4 \%)$, yet a very systematic variation, which represents the ratio of the efficiencies of modules 4 and 5. This was

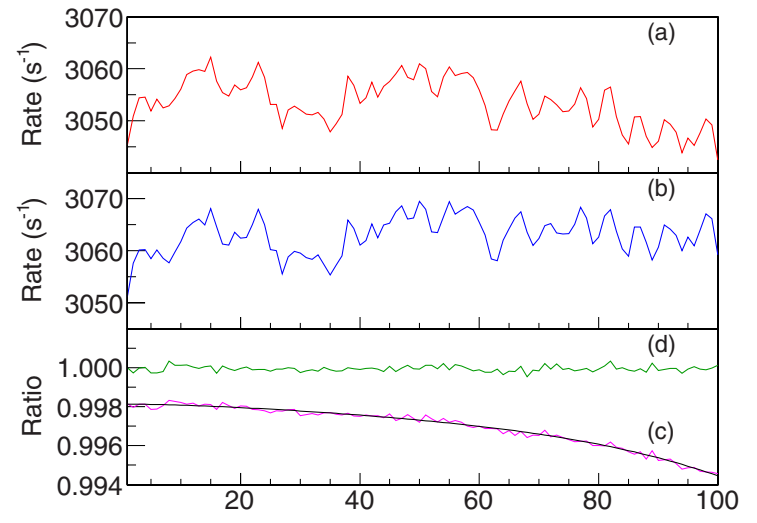

Figure 2. Muon rates for the telescope modules, (a) 4, (b) 5 for 100 days (1 Jan.-10 Apr. 2006. Ratio of the muon rates, (c) before efficiency correction, and a fourth order polynomial fit, (d) after the efficiency correction.

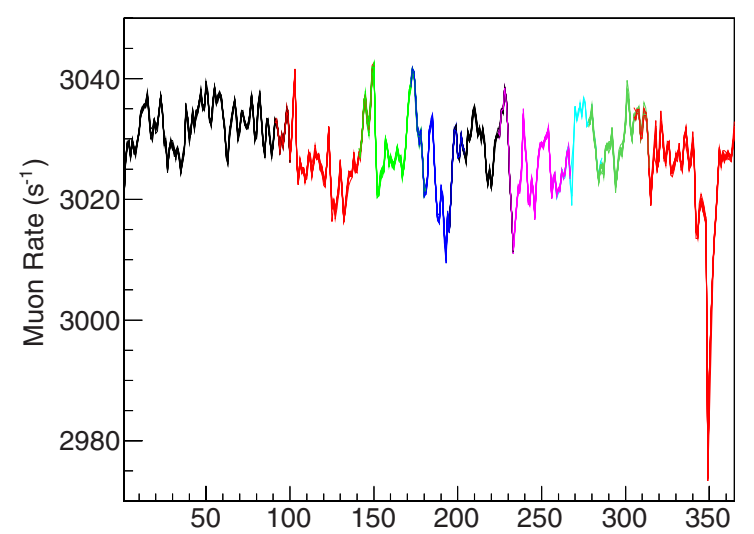

Figure 3. Muon rates for $364.1 \mathrm{~d}$ for sixteen telescope modules from 1 Jan.-30 Dec. 2006 after efficiency correction modeled by a fourth order polynomial.

caused by a gradual change in the detection efficiency of the PRCs which was modeled by using a fourth order polynomial in time. Since the GRAPES-3 muon telescope consists of sixteen modules, a total of 120 independent combinations of efficiency ratios could be formed using the corresponding fourth order polynomials (for sixteen modules) for daily data. The 120 series of 365 ratios for the year 2006 represent the ratios of their unique efficiency profiles of the corresponding modules. Since the magnitude of this variation is rather small $(<1.0 \%)$, this problem could be readily solved after performing a Taylor series expansion, and by using the singular value decomposition (SVD) technique which yielded the five coefficients corresponding to each fourth order polynomial describing the time-dependent efficiencies of each of the sixteen modules. The details of this technique are described elsewhere [18]. The efficiency ratios for modules 4 and 5 obtained by SVD technique yielded a fit which described the data very well as is seen from Fig. 2c. When the muon rates for modules 4 , and 5 were corrected by using the corresponding efficiency variations, the resultant ratios shown in Fig. $2 \mathrm{~d}$ almost equal unity as is expected for an ideal correction.

In Fig. 3 the daily rates of all modules are shown for the whole year (2006) after correcting for efficiency variations using the fourth order polynomial correction. Except for an Fd event in December 2006, the muon rates displayed 


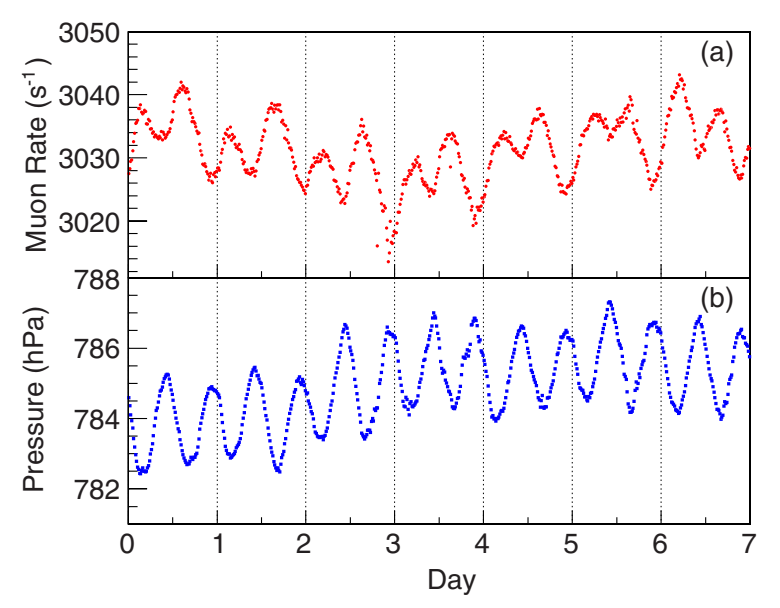

Figure 4. Variation of, (a) mean muon rate, (b) pressure (hPa) for the week 1-7 March 2006 [20].

a narrow variation of only 20 (3010-3040) counts for the rest of the year. Also, the sixteen rates are nearly indistinguishable from each other. The rms spread in the daily muon rates was only $0.32 \mathrm{~s}^{-1}$ which was comparable to the statistical error of $0.26 \mathrm{~s}^{-1}$. Thus, the efficiency correction was fairly effective, and the rms spread of $0.32 \mathrm{~s}^{-1}$ in a rate of $3000 \mathrm{~s}^{-1}$ implies a precision of $0.01 \%$ in the measured relative efficiencies.

\subsection{Pressure correction}

The muons detected by GRAPES-3 are produced by GCRs of energy $\sim 100 \mathrm{GeV}$, and are modulated by the solar activity, and therefore, display a $24 \mathrm{~h}$ periodic variation due to the rotation of the Earth. However, at near-equatorial locations such as the GRAPES-3 [2] also show a $12 \mathrm{~h}$ periodic variation in addition to the $24 \mathrm{~h}$ variation of solar origin. The $12 \mathrm{~h}$ period is induced by the heating of the upper atmosphere which results in a non-linear response of the atmosphere with a dominant $12 \mathrm{~h}$ oscillation in pressure at near-equatorial locations (amplitude $\sim 1 \mathrm{hPa}$ ) [19].

Since variations in atmospheric pressure change the total mass of the air column above the telescope, it causes a corresponding change in the rate of muons which was observed as an anti-correlation of the muon rate with pressure. The measured muon rate variation is a combined effect of the change induced by the pressure and the solar activity. A correction for the pressure variation can be implemented by using the pressure coefficient $\beta$ which can be obtained from the measured muon rate. The pressure at Ooty displays a dominant $12 \mathrm{~h}$ periodic variation as shown in Fig. 4a for an interval of one week. This variation produces a synchronous response in the muon rate due to an inherent anti-correlation of these two variables. This anti-correlation was studied by obtaining the fast Fourier transform (FFT) of the two sets of data. Since the use of FFT requires an uninterrupted time series of $2^{N}$ intervals, where $\mathrm{N}$ is a positive integer, the muon rates were converted into an uninterrupted stream of $2^{17}=131072$, 4 min intervals covering $364.01 \mathrm{~d}$ starting from 1 January 2006. The deviation in muon rate from its mean was calculated in percent, and an FFT performed on muon rate as well as on the pressure data. By using a narrow band filter centered at a period of $12 \mathrm{~h}$ the FFT power from the muon and pressure data were extracted. These power

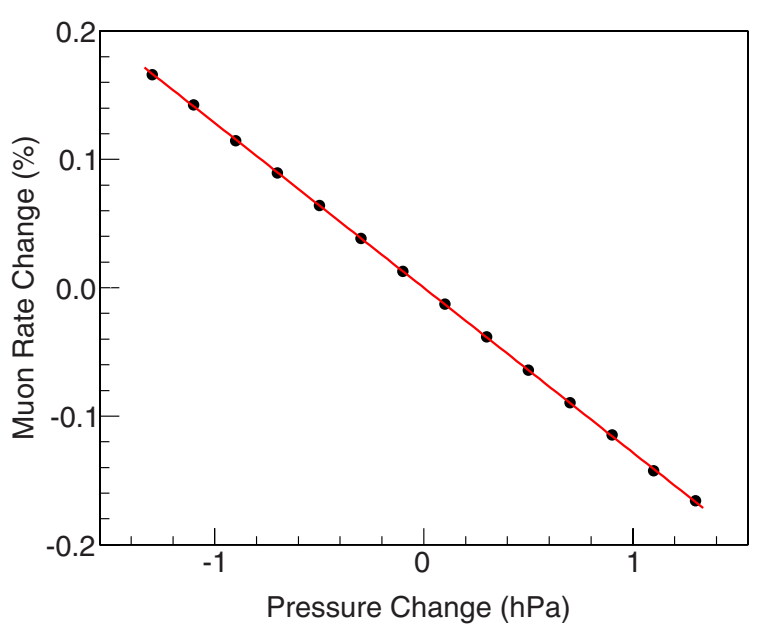

Figure 5. Dependence of muon rate on atmospheric pressure relative by IFFT [20].

spectra permitted the segregation of the $12 \mathrm{~h}$ barometric effect in the muon data from all other phenomena. Next by taking the inverse fast Fourier transform (IFFT) of the two filtered data sets, the muon, and pressure data were converted back into time domain [20].

In Fig. 5 the IFFT muon data are plotted against corresponding pressure data. The error on each data point is invisible because of its small value. A linear fit shown by the solid line yielded a pressure coefficient $\beta$ of $-0.128 \% \mathrm{hPa}^{-1}[20]$.

\subsection{Temperature correction}

Atmospheric temperature undergoes annual variations due to the Earth's orbital movement around the Sun. This causes an annual variation in the atmospheric density, resulting in a similar modulation in the measured muon rate. This annual modulation was first reported over six decades ago. A phenomenological framework was also sketched by defining the concept of an effective temperature ' $\mathrm{T}_{\text {eff' }}$ ' $\mathrm{T}_{\text {eff }}$ can be calculated by using the thermal profile of the upper atmosphere. The significance of $\mathrm{T}_{\text {eff }}$ is that a real atmosphere behaves exactly as an isothermal atmosphere at a fixed temperature of $\mathrm{T}_{\text {eff }}$. However, the calculation of $\mathrm{T}_{\text {eff }}$ requires as input the attenuation length, $\lambda$, of the hadron in the atmosphere [21]. Due to its near-equatorial location $\left(11.4^{\circ} \mathrm{N}\right)$ the upper atmospheric temperature at Ooty shows a relatively small annual variation $(\sim 1 \mathrm{~K})$. The $\mathrm{GeV}$ muons recorded by GRAPES-3 telescope are produced by the GCRs of average energy $<100 \mathrm{GeV}$. The mesons produced by the GCRs in the atmosphere have energies of 10 s of $\mathrm{GeV}$ which decay in the upper atmosphere into $\mathrm{GeV}$ muons which are detected by the GRAPES-3 telescope. The energy loss of the muons along a direction $\theta$ is $\leq 2 \sec (\theta)$ which is comparable to their energy. This results in the decay of a significant fraction of the muons before being detected by the GRAPES-3. When the temperature of the atmosphere decreases, the fraction of the muons decaying also decreases due to reduced length traveled due to thermal contraction of the atmosphere. This results in muons displaying an anti-correlation with the temperature which was measured by using the GRAPES-3 data collected over six years (2005-2010). The anti-correlation 


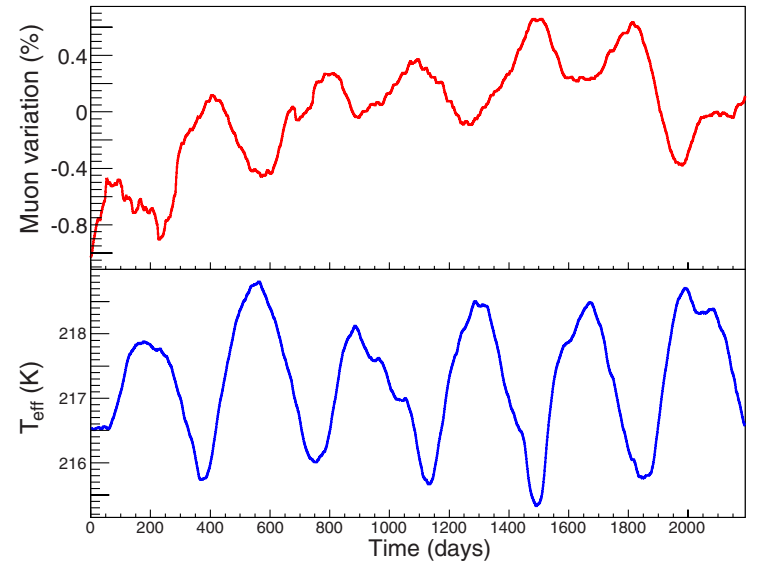

Figure 6. Variation of (a) muon rate, (b) $\mathrm{T}_{\text {eff }}(\mathrm{K})$ [22].

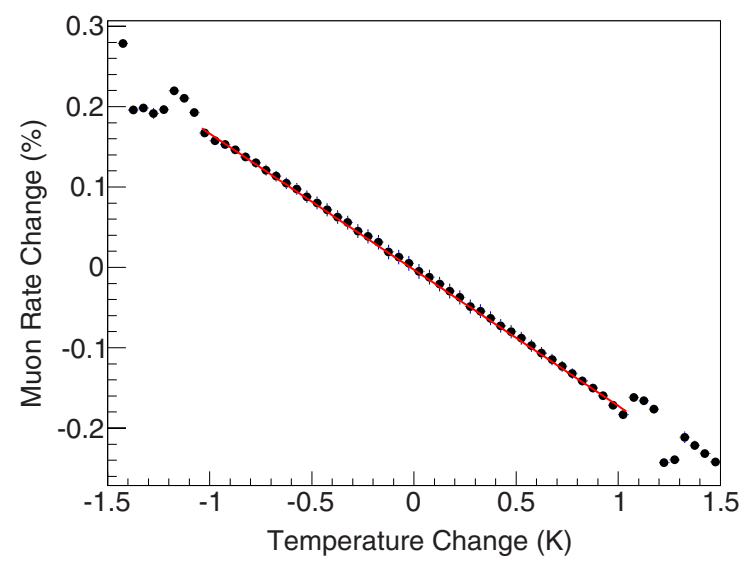

Figure 7. Variation in muon intensity as a function of $\Delta \mathrm{T}$ relative to respective mean values [22].

between the annual variation in $\mathrm{T}_{\text {eff }}$ and the muon rate was used to measure the temperature coefficient $\alpha_{\mathrm{T}}$ by the fast Fourier transform (FFT) technique described in [20].

During the six year interval (2005-2010), relatively faster variations caused by solar phenomena such as the Forbush decreases, the $27 \mathrm{~d}$ solar rotation etc. were removed by using an effective low-pass filter created by the running average over a window of $60 \mathrm{~d}$. The resultant percent deviation in the muon rate, and $\mathrm{T}_{\text {eff }}$ are shown in Fig. 6a, and Fig. 6b, respectively. By using the FFT technique as outlined above in $\S 2.2$, and a narrow-band filter centered at a frequency of 1 per year the dependence of the muon rate on $\mathrm{T}_{\text {eff }}$ was obtained as shown in Fig. 7.

For an attenuation length $\lambda=120 \mathrm{~g} \mathrm{~cm}^{-2}$ the value of the temperature coefficient, $\alpha_{\mathrm{T}}$, was found to be $(-0.169 \pm 0.001) \% \mathrm{~K}^{-1}$. It was also found that the magnitude of $\alpha_{\mathrm{T}}$ scaled with the assumed value of $\lambda$ in the range $\lambda=80-180 \mathrm{~g} \mathrm{~cm}^{-2}$. However, the actual magnitude of the correction to the muon rate was independent of the $\lambda$ value used, indicating the intrinsic robustness of the result [22].

\section{GRAPES-3 results}

\subsection{Atmospheric acceleration}

It was observed that thunderstorm activity is accompanied by sudden changes in the rate of detected muons over a time scale of $\sim 30$ minutes. However, this phenomena

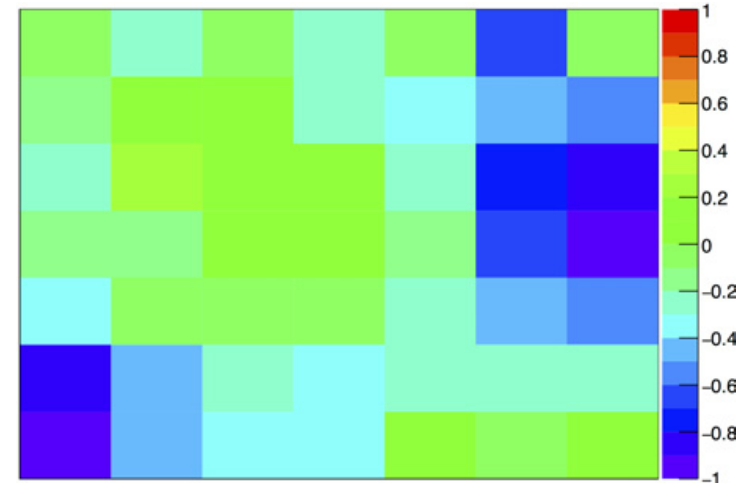

Figure 8. $7 \times 7$ panels display variation of muon rate along 49 directions on 30 September 2015 from 17:53 to 18:20 h (IST) during a thunderstorm. A significant deficit is seen in NE, and SW directions for 27 minutes.

is not observed in the muon rate in all directions, but is confined to a limited solid-angle. In Fig. 8 color coded reduction in the rate of muons in percent is shown along 49 directions constituting the GRAPES-3 field of view (FOV) of $2.3 \mathrm{sr}$. This event was observed during a massive thunderstorm on 30 September 2015 which lasted for 27 minutes. A significant deficit may be seen in NE, and SW directions.

During this event the reduction in the muon rate was accompanied by the presence of a large electric field that was recorded by the GRAPES-3 electric field monitors (EFMs) on ground. The polarity of the electric field indicated that the ground was at a large positive electric potential relative to the upper atmosphere. The variation in the rate of muons during 17:53 to 18:20 h Indian Standard Time (IST) on 30 September 2015 in the GRAPES-3 FOV is shown in Fig. 8 where a large reduction in the rate of muons is clearly seen in the NE and SW directions.

The GRAPES-3 EFMs are distributed widely, with a typical distance of $\sim 3 \mathrm{~km}$ between them. During this event all four EFMs showed positive electric potential on ground which was concurrent with the deficit seen in the muon rate. It is well-known that the ratio of the $\mu^{+}$to $\mu^{-}$is $>1.0$ at energies of a few $\mathrm{GeV}$ which is known to gradually increase at higher energies [23-25].

This phenomena can be understood when a cloud in the upper atmosphere is negatively charged. This would result in deceleration of $\mu^{+}$and acceleration of $\mu^{-}$. Since their ratio is greater than one the rate of muons, which is a sum of both polarities, will show a net reduction. The GRAPES-3 muon telescope does not operate with magnetic field so it is not possible to distinguish the two types of muons. At present we are carrying out large-scale Monte Carlo simulations by using CORSIKA [26] to generate the muons in the atmosphere which are subsequently detected by the muon telescope. We had also modified CORSIKA to include the presence of electric potential, which leads to the acceleration of these muons. In Fig. 9 the percentage change in the muon rate is shown as a function of applied electric potential [27]. It is to be noted that when a positive electric potential relative to the ground is applied, the muon rate initially decreases slowly up to $1 \mathrm{GV}$, and thereafter more rapidly. However, when a negative potential is applied then the muon rate initially increases slowly up to $0.8 \mathrm{GV}$, and thereafter decreases 


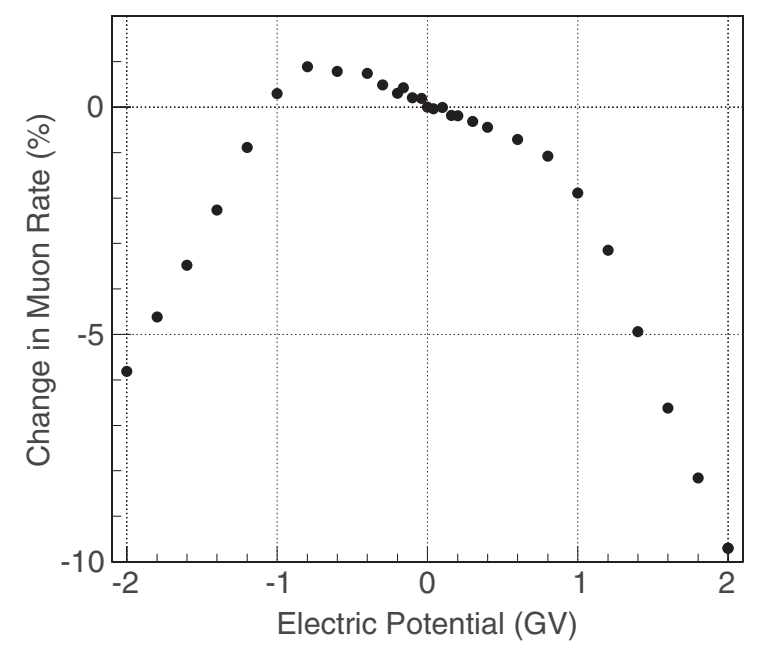

Figure 9. Variations in muon rate due to an applied electric potential in the upper atmosphere for intervals of $100 \mathrm{MV}$ during a thunderstorm.

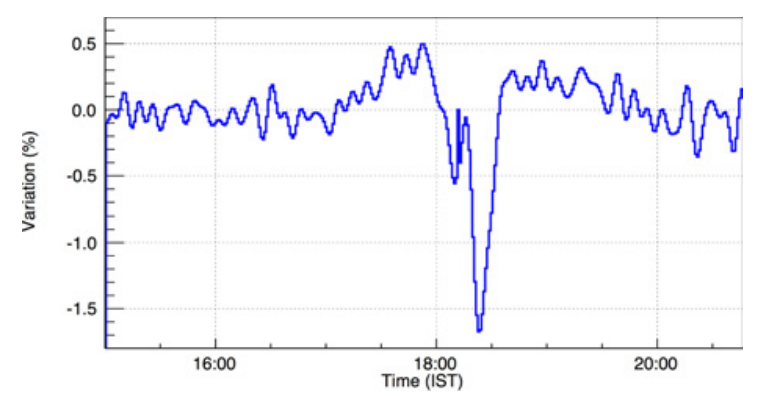

Figure 10. Percent variations in muon rate observed during the thunderstorm on 30 September 2015 during interval 15-21 h IST. An electric potential of $1.2 \mathrm{GV}$ is required to produce observed maximum decrease of $1.7 \%$.

rapidly. The muon rates for the nine NE, and five SW directions were combined, and are shown in Fig. 10 as a function of time for the 30 September 2015 thunderstorm event. The observed maximum decrease of $1.7 \%$ in the rate of muons shown in Fig. 10 requires an electric potential of nearly $1.2 \mathrm{GV}$ to be present during this thunderstorm.

\subsection{Detection of a GCR burst}

The GCRs producing the low-energy $(\mathrm{GeV})$ muons detected by the GRAPES-3 telescope are affected by the geomagnetic field, and the Monte Carlo simulations can accurately account for it. Depending on the location on Earth, the geomagnetic field causes a deflection of the incoming GCR in the surrounding space. Since the GCRs are overwhelmingly positive particles, the minimum energy per unit charge, also called the cutoff rigidity has a lower value from the west when compared to the east direction. This geomagnetic deflection has a significant effect on the muons detected by GRAPES-3 due to the location of Ooty close to the geomagnetic equator. To account for the deflection of the GCRs in the Earth's magnetic field, a model of the geomagnetic field, namely, "International Geomagnetic Reference Field 11th generation model (IGRF-2011)" [28] was employed.

The characteristics of the muons detected, such as the energy spectrum and the angular distribution are governed by the interaction properties of GCRs producing

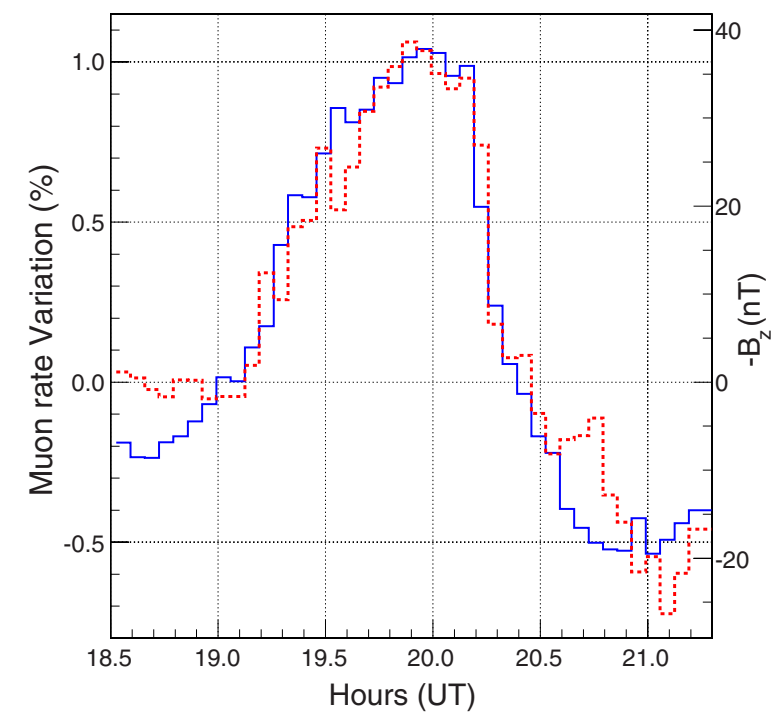

Figure 11. Muon-rate (solid-line), and $-\mathrm{B}_{\mathrm{z}}$ (broken-line) on 22 June 2015, correlation coefficient $\mathrm{R}=-0.94$ from [30].

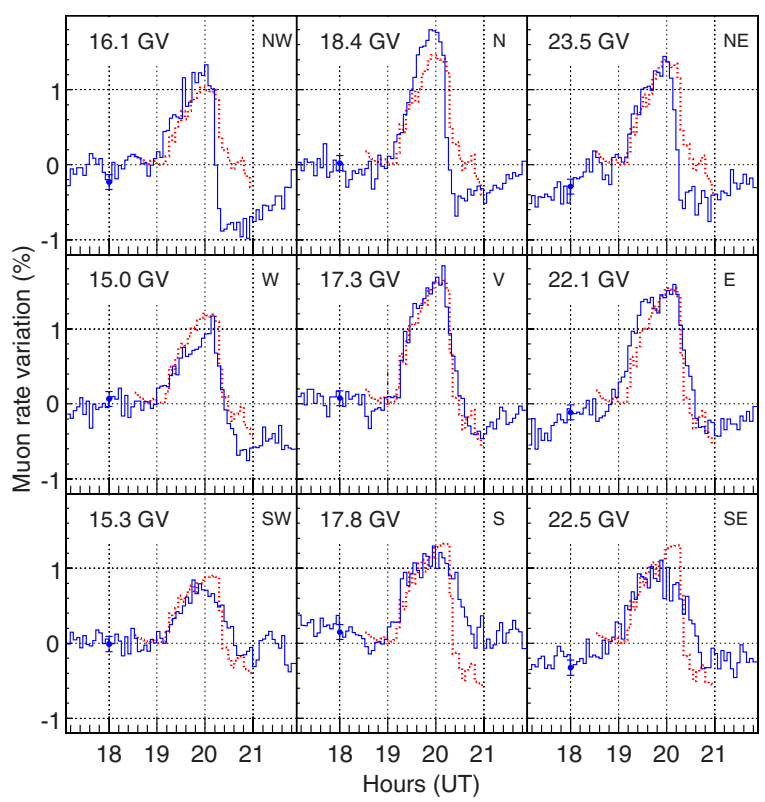

Figure 12. Muon-rate variations in nine directions observed by GRAPES-3 on 22 June 2015 shown by solid-line. Simulation results normalized to data by scaling the IMF 17 times shown by the broken-line. Cut-off rigidities (GV), and error bars are shown for each direction [30].

them. The angular distribution of the muons is a consequence of several effects which include attenuation in the atmospheric, the hadronic cross sections of parent particles, their production heights, particle multiplicities, and their pseudo rapidity distributions. These interaction properties are parametrized in a hadronic interaction generator based on specific assumptions which include extrapolation of various properties from accelerator based measurements.

Simulations were carried out using CORSIKA [26], and FLUKA [13] to describe low-energy, and SIBYLL [15] for high-energy hadronic interactions for protons as GCRs with a differential spectral slope of -2.7 near the top of the atmosphere. The trajectory of a proton (p) was simulated using back-tracing methods by launching 


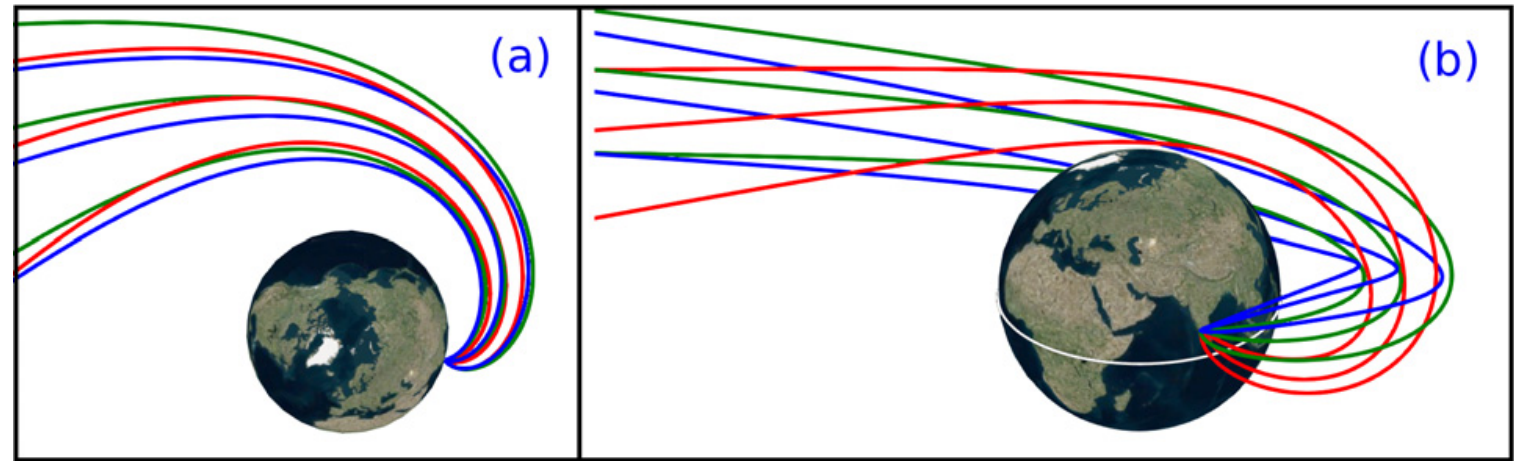

Figure 13. Trajectories of the GCRs near cutoff rigidity viewed from, (a) north pole, (b) equator. NW, N, NE shown in blue, W, V, E in green, SW, S, SE in red [30].

an anti-proton $(\overline{\mathrm{p}})$ of the same energy in the same direction into space from the GRAPES-3 muon telescope. If the $\bar{p}$ trajectory escapes into space without striking the Earth, then a ' $p$ ' of the same energy would be able to reach the Earth from outer space from the same direction. The minimum energy of $\bar{p}$ escaping into space defines the cutoff rigidity of a $\mathrm{p}$ in that direction by the use of this "back tracing" method. Protons above the geomagnetic cutoff rigidity were subjected to the deflection in the geomagnetic field by using a cosmic ray trajectory program [29]. For this purpose, $10^{9}$ GCR protons above $10 \mathrm{GeV}$ were simulated. The muons above the $1 \mathrm{GeV}$ threshold were tracked down to the location of GRAPES-3. Thereafter, each muon incident at a zenith angle $\theta$ was tested if it crossed the detection threshold of $\sec (\theta) \mathrm{GeV}$. The muons satisfying this cut were registered as detected. The resultant hits in the four layers of PRCs were histogrammed into nine bins, namely NE, E, SE, N, $\mathrm{V}, \mathrm{S}, \mathrm{NW}, \mathrm{W}, \mathrm{SW}$ as was done for real data. $10^{9}$ incident GCR protons resulted in detection of $2 \times 10^{8}$ simulated muons.

The GRAPES-3 had relatively high cutoff rigidities (15-24 GV) along the nine directions covering a solid angle of 2.3 sr. On 22 June 2015 18:40 UT a coronal mass ejection $(\mathrm{CME})$ reached the Earth and triggered a severe G4-class geomagnetic storm, hereafter 'storm'. Starting at 19:00 UT, the muon telescope registered a $2 \mathrm{~h}$ duration burst of GCRs that showed a strong $94 \%$ correlation with a spike in the interplanetary magnetic field (IMF) of $40 \mathrm{nT}$ as shown in Fig. 11 [30].

Simulations carried out to explain the occurrence of this burst to be due to the change in the cutoff rigidities caused by the arrival of the IMF showed that a large $(17 \times)$ compression of the IMF to $680 \mathrm{nT}$ which led to the lowering of the cutoff rigidities could generate this burst as shown in Fig. 12. Here, the value of $680 \mathrm{nT}$ represented a short-term change in the geomagnetic field around the Earth, averaged over seven times its volume [30].

Due to the lowering of the cutoff rigidities the GCRs were deflected from the day-side of the Earth to the nightside by $210^{\circ}$ in longitude as shown in Fig. 13 from polar, and equatorial views. This offered a natural explanation of the night-time detection of the burst by the GRAPES-3 muon telescope. The simultaneous occurrence of the burst in all directions pointed to its origin close to the Earth possibly within the magnetosphere. Detection of this burst indicated a transient weakening of the Earth's magnetic shield. A study of these events may provide a better understanding of future solar induced storms that could have devastating impact on the modern technological infrastructure on the Earth [30].

As part of the expansion of the muon telescope, a total of 3780 large proportional counters (PRCs) each $600 \times$ $10 \times 10 \mathrm{~cm}^{3}$ in size, are required. Out of these 2500 PRCs $(65 \%)$ have already been fabricated and tested. About $20 \%$ of the total PRCs have been deployed in the field. The ongoing expansion activity for enhancing the sensitivity of the GRAPES-3 muon telescope by doubling its area is expected to be completed in a years time.

\section{Summary}

Measurements using the high efficiency tracking muon telescope in the GRAPES-3 EAS array have been used to observe the acceleration of muons in large-scale electric fields during powerful thunderstorms. By exploiting the high statistics GRAPES-3 muon data, the relative efficiencies of the sixteen modules of the GRAPES-3 muon telescope could be accurately determined. By the application of the fast Fourier transform technique corrections to the muon rate due to variations in the atmospheric pressure, and the atmospheric temperature could be accurately measured in a fairly robust manner. These corrections paved the way for the discovery of transient weakening of the Earth's magnetic shield during a severe G4-class geomagnetic storm. The ongoing major expansion activity for enhancing the sensitivity of the GRAPES-3 muon telescope by doubling its area is expected to be completed in a years time.

We thank the members of the GRAPES-3 collaboration who were instrumental in obtaining these exciting results discussed here.

\section{References}

[1] S.K. Gupta et al., Nucl. Instr. Meth. A 540, 311 (2005)

[2] Y. Hayashi et al., Nucl. Instr. Meth. A 545, 643 (2005)

[3] D.M. Smith, Science 307, 1085 (2005)

[4] M.S. Briggs, AIP Conf. Proc. 1366, 47 (2011)

[5] https://science.nasa.gov/science-news/ science-at-nasa/2014/31dec_tgfs

[6] T. Nonaka et al., Phys. Rev. D 74, 052003 (2006)

[7] P. Subramanian et al., Astron. Astrophys. 494, 1107 (2009) 
[8] K.P. Arunbabu et al., Astron. Astrophys. 555, A139 (2013)

[9] K.P. Arunbabu et al., Astron. Astrophys. 580, A41 (2015)

[10] S.K. Gupta et al., Phys. Rev D 68, 052005 (2003)

[11] H. Fesefeldt, Report PITHA-85/02 RWTH Aachen (1985)

[12] H. Petersen, et al., Phys. Rev. C 78, 044901 (2008)

[13] http://www.fluka.org

[14] S. Ostapchenko, Phys. Rev. D 83, 014018 (2011)

[15] R.S. Fletcher et al., Phys. Rev. D 50, 5710 (1994)

[16] T. Pierog and K. Werner, Nucl. Phys. B Proc. Suppl. 196, 102 (2009)

[17] H. Tanaka et al., J. Phys. G: Nucl. Part. Phys. 39, 025201 (2012)

[18] P.K. Mohanty, Ph.D. Thesis Unpublished (2014)
[19] R.S. Lindzen and S. Chapman, Space Sci. Rev. 10, 3 (1969)

[20] P.K. Mohanty et al., Astropart. Phys. 79, 23 (2016)

[21] P. Barrett et al., Rev. Mod. Phys. 24, 133 (1952)

[22] K.P. Arunbabu et al., Under preparation (2017)

[23] S. Haino et al., Phys. Lett. B 594, 35 (2004)

[24] P. Achard et al., Phys. Lett. B 598, 15 (2004)

[25] P. Adamson et al., Phys. Rev. D 76, 052003 (2007)

[26] D. Heck et al., CORSIKA: A Monte Carlo code to simulate EAS, 6019 (1998)

[27] B. Hariharan et al., Proc. of Science PoS (ICRC2015) 448 (2015)

[28] C.C. Finlay et al., Geophys. J. Int. 183, 1216 (2010)

[29] D.F. Smart et al., Space Sci. Rev. 93, 305 (2000)

[30] P.K. Mohanty et al., Phys. Rev. Lett. 117, 171101 (2016) 